\title{
Original
}

\section{Valoración de las interconsultas realizadas a un servicio de urologia, a nivel hospitalario}

\author{
Patricio Navarro Medina, Antonio Blanco Diez, Alejandro Alvarado Rodriguez, \\ Eduardo Barroso Deyne, José Luis Artiles Hernández, Nicolás Chesa Ponce \\ Complejo Hospitalario Univ. Insular Materno Infantil de Gran Canaria. Las Palmas de Gran Canaria, España
}

\begin{abstract}
Resumen
Objetivo: Los objetivos principales de este estudio fue valorar la calidad de la información contenida, las características de las interconsultas (IC) y así como conocer la actuación realizada por el servicio de Urología en nuestro hospital.

Material y métodos: Se realizó un estudio descriptivo observacional donde fueron analizados los documentos de IC recogidos por el servicio de Urología durante 8 meses. Se utilizaron criterios de calidad previamente definidos por Irazábal et al (modificado) y Batista Miranda JE et al.

Resultados: Fueron recogidos un total de 411 IC, llegadas al servicio de forma consecutiva. Procedentes de 27 servicios hospitalarios. El $82,2 \%$ de los pacientes derivados fueron varones. La edad media de los pacientes fue de 63,25 años. 192 pacientes tenían antecedentes personales urológicos de interés (47\%). Las IC se agruparon en 27 motivos diferentes. El motivo de consulta más frecuente fue el incidentaloma con 21,5\% . Respecto al grado de cumplimiento del documento de IC: había constancia de datos de filiación (96,3\%), una descripción de la sintomatología adecuada en el 68,2\% y la exploración física aparecía reflejada en el 8,8\% del total de las IC. Fueron solicitadas como IC normales el 63,4\%, preferentes 25\% y urgentes 11,7\%. Los días de respuesta a las IC fueron de media de 1,45 días con desviación típica de 1,65 días. Según los criterios establecidos, para clasificar los documentos por nivel de adecuación, fueron catalogadas como inapropiadas al $32,9 \%$ de las IC ( $n=135)$. Fueron clasificadas en grado 1 el 44,6\% ( $=183$ ), grado 2 el 20\% ( $n=82$ ) y grado 3 el 2,4\% (n=10). La actitud que se tomó: se remitió a Consultas Externas de Urología al 10,5\% del total de las IC, al Centro de Atención Especializada al 7,8\%, se solicitó pruebas complementarias al 7,8\%, se remitió al Pruebas funcionales de Urología, para la realización de alguna prueba complementaria a 61 pacientes (14,9\%), se presentó en Sesión Clínica de Urología al,6\% de los pacientes, se trasladó al Servicio de Urología a 2 pacientes (0,5\%) y se tomó alguna otra actitud terapéutica en el 19,6\%, estos resultados tuvieron una significación de p=0,000.

Conclusiones: En nuestro estudio, observamos un alto porcentaje en cuanto a la constancia de los datos de filiación ligeramente inferior en la descripción de la sintomatología y considerados bajos en la exploración física. Un alto porcentaje de IC consideradas inapropiadas. Respecto a la actitud que tomó el Servicio de Urología, quizás hubo una infrautilización de la Unidad de Pruebas funcionales. Hasta el momento y bajo nuestro conocimiento sólo existe un estudio realizado en el ámbito de las IC realizadas al Servicio de Urología en un Hospital de tercer nivel y creemos que la realización de estudios mejoraría la calidad de la asistencia a los pacientes.
\end{abstract}

Palabras clave: Interconsultas. Urología. Hospitalaria.

\section{Evaluation of the consultants to a urology service at hospital level}

\section{Abstract}

Summary: Objective: The main objectives of this study were to value the quality of the contained information, the characteristics of the consultants (IC) and as well as to know the performance carried out by the service of Urology in our hospital.

Material and methods: It was carried out an observational descriptive study where the documents of IC picked up by the service of Urology during 8 months were analyzed. Previously defined approaches of quality were used by Irazábal et al (changed) and Batista Miranda JE et al.

Results: They were picked up a total of 411 IC, arrivals to the service in a serial way. Coming from 27 hospital services. $82,2 \%$ of the derivatived patients were male. The half age of the patients was of 63, 25 years. 192 patients had personal urological records of interest (47\%). The IC grouped in 27 different reasons. The reason of more frequent consultation was the incidentaloma with $21,5 \%$. Regarding the grade of execution of the document of IC: there was perseverance of filiation data (96,3\%), a description of the symptoms was in $68,2 \%$ and the physical examination appeared reflected in $8,8 \%$ of the total of the IC. They were requested as normal IC $63,4 \%$, preferent $25 \%$ and urgent $11,7 \%$. The days of answer to the IC it was of stocking of 1,45 days with typical deviation of 1,65 days. According to the established approaches, to classify the documents for adaptation level, they were classified as inappropriate to 32,9\% of the IC (n=135). They were classified in grade 1 the 44,6\% ( $n=183)$, grade $220 \%(n=82)$ and grade $32,4 \%(n=10)$. The attitude that was taken: it was remitted to External Consultations of Urology to $10,5 \%$ of the total of the IC, to the Center of Specialized Attention to $7,8 \%$, it was requested complementary tests to $7,8 \%$, it was remitted to the functional Tests of Urology, for the realization of some complementary test to 61 patients ( $14,9 \%$ ), it was presented in Clinical Session of Urology to $5,6 \%$ of the patients, he/she moved to the Service of Urology to 2 patients (0,5\%) and he/she took some other therapeutic attitude in $19,6 \%$, these results had a $\mathrm{p}=0,000$ significance.

Conclusion: In our study, we observe a high percentage as for the perseverance of the data of lightly inferior filiation in the description of the symptoms and considered low in the physical exploration. A high percentage of inappropriate considered IC. Regarding the attitude that took the Service of Urology, maybe had a low used of the Functional Unit. Until the moment and under our knowledge a study carried out in the environment from the realized IC to the Service of Urology in a Hospital of third level only exists and we believe that the realization of studies would improve the quality from the attendance to our patients.

Keywords: Consultants. Urology. Hospital. 
$\mathrm{E}^{\mathrm{s}}$ sistema actual de relación entre los diversos servicios asistenciales hospitalarios se fundamenta en la interconsulta (IC) médica. Esta actividad es de gran importancia para el correcto funcionamiento de los diferentes servicios, ya que cumple la función de aportar conocimientos específicos a otras especialidades no familiarizadas con la causa de la IC correspondiente. Sin embargo, puede suponer una elevada carga de trabajo para el servicio al que se solicita. A pesar de su interés, pocos trabajos la han estudiado con una profundidad suficiente y en el caso concreto de la Urología, y bajo nuestro conocimiento sólo ha sido publicado los resultados de una serie de pacientes ${ }^{1}$. En nuestro país los trabajos publicados se centran en la interrelación entre Atención Primaria y Especializada ${ }^{5}$.

No obstante, tenemos la impresión de que la comunicación entre ambos en nuestro medio es deficiente. Siendo diversos elementos los posibles causantes de la descoordinación en el sistema: déficit de recursos, tiempo, sobrecarga asistencial, fallos en el canal de comunicación y falta de respuesta del médico consultado.

Los objetivos principales de este estudio fue valorar la calidad de la información contenida y las características de las interconsultas, así como conocer la actuación realizada por el servicio de Urología en nuestro hospital. Como objetivos secundarios nos propusimos conocer las características demográficas de la población, describir la distribución de las especialidades demandantes, el carácter preferencial del documento, la frecuencia de la misma y el tipo de patología atendida.

\section{MATERIAL Y MÉTODOS}

Se trata de un estudio descriptivo observacional donde fueron analizados los documentos de interconsulta, llegados al Servicio de Urología. Procedentes de 27 servicios (10 quirúrgicos y 17 médicos), del Complejo Hospitalario Universitario Insular Materno Infantil de Gran Canaria, con pacientes mayores de 14 años que consultaron durante 9 meses.

El circuito habitual que sigue el documento de interconsulta en nuestro hospital es el siguiente: el médico consultor realiza la IC y lo entrega a la enfermero/a o supervisor/a de enfermería quién lo entrega íntegro a un/a celador/a y éste lo lleva en mano a la secretaría del servicio consultado (en este caso Urología). En nuestro servicio no mantiene a un urólogo, con dedicación exclusiva a la resolución de
IC. Pero si mantiene a un urólogo de forma permanente localizado, por lo que las IC urgentes pueden ser solicitadas por este medio o a través del buscapersonas, con una respuesta inmediata. Por lo que, las IC procedentes del Servicio de Urgencias y un porcentaje importante de las realizadas desde los pacientes hospitalizados en las plantas, no están reflejados en el presente estudio.

Las variables recogidas en el documento de interconsulta para valorar el cumplimiento y el nivel de calidad del mismo, así como para conocer las características demográficas de la población derivada fueron las siguientes: edad del paciente, sexo, especialidad que la realiza, carácter con el que se requiere la contestación (urgente, preferente o normal, filiación del paciente, referencia a antecedentes personales urológicos de interés, referencia a medicación habitual, referencia a sintomatología, referencia a exploración física, referencia a medidas terapéuticas para el proceso actual, motivo de la consulta. Para la valoración de la calidad se utilizaron criterios de calidad previamente utilizados en otros estudios Rubio et al. ${ }^{2}$ (Tabla 1) modificándolas parcialmente y las aportaciones realizadas por Batista Miranda JE et al. ${ }^{3}$ (Tablas 1 y 2). Por otro lado, fueron analizados las variables correspondientes a nuestro servicio; días de respuesta, decisión tomada por el urólogo (p.ej: ajuste de tratamiento, remisión a consultas externas de urología, pruebas funcionales de urología, etc).

En la evaluación, los Urólogos, resto de facultativos así como personal sanitario como administrativo desconocían que se estaba realizando la recogida de los documentos.

Tabla 1. Criterios en el cumplimiento del documento de interconsulta

1. Constancia de datos de filiación

2. Legibilidad

3. Referencia a antecedentes personales

4. Referencia a medicación habitual

5. Referencia a sintomatología

6. Referencia a exploración física

7. Referencia a pruebas complementarias

8. Referencia a medidas terapéuticas para el proceso actual

9. Figura diagnóstico de presunción o diagnóstico diferencial

10. Motivo de la consulta o derivación

Modificada de Rubio et al. 
Tabla 2. Criterios de evaluación

Grado 0: Inapropiadas (no se toman actitudes terapéuticas, no se remite a Consultas Externas, no se remite a Pruebas Funcionales de Urología ni se solicitan pruebas complementarias).

Grado 1: Consultas realizadas por otros especialistas en referencias a problemas urológicos concretos (pe: elevación del PSA).

Grado 2: Consultas que precisaban ser resueltas en las siguientes horas (pe: sondaje).

Grado 3: Consultas urgentes (p.ej.:derivación urinaria en paciente séptico)

Batista Miranda JE et al.

Para el análisis de los datos del estudio se utilizó el programa informático SPSS en su versión 11.5.

\section{RESULTADOS}

Fueron recogidos un total de 411 documentos de interconsultas, llegadas al servicio de forma consecutiva. Procedentes de 27 servicios (10 quirúrgicos y 17 médicos), de las cuales 309 IC de servicios médi$\cos (27,6 \%$ procedían del servicio de Medicina Interna) y 101 de servicios quirúrgicos (14,4\% procedentes del servicio de Cirugía General y Digestiva).

El 18,8\% de los pacientes derivados fueron mujeres $(n=77)$. La edad media de los pacientes remitidos fue de 63,25 años con una desviación típica de 17,11 años (máximo 98 años y mínimo 15 años). 192 pacientes tenían antecedentes personales urológicos de interés (47\%). De estos el 58,6\% eran mayores de 65 años. Sin haber diferencias significativas entre servicios médicos y quirúrgicos. $47,6 \%$ y $42,6 \%$, respectivamente $(\mathrm{p}=0,003$ ).

Las interconsultas se agruparon en 27 motivos diferentes. El motivo de consulta más frecuente fue el incidentaloma con 21,5\% (los hallazgos más frecuentemente encontrados fueron la hidronefrosis por ecografía y TAC, lesión vesical o lesión renal), problemas relacionados con el sondaje 19,2\%, hematuria el 13,7\% y PSA elevado en el $9,8 \%$. En éstos últimos, el $68,4 \%$, eran mayores de 65 años. Que fueron contestadas por 8 urólogos o residentes de urología, el 74,8 \% de las IC fueron realizadas por los segundos.

Respecto al grado de cumplimiento del documento de intercon- sulta, había constancia de datos de filiación (96,3\%), una descripción de la sintomatología adecuada en el $68,2 \%(76,1 \%$ de los servicios médicos y servicios quirúrgicos en el 50,8\% p=0,000). La exploración física aparecía reflejada en el 8,8\% del total de las IC, sin diferencias significativas entre servicios médicos ni quirúrgicos. Y ya se habían solicitado las pruebas complementarias necesarias al $21 \%$.

Fueron solicitadas como IC normales 260 $(63,4 \%)$, preferentes $102(25 \%)$ y urgentes 48 $(11,7 \%)$, no han sido reflejadas las IC realizadas a los buscas (residentes y adjuntos), los pacientes remitidos desde urgencias, y IC realizadas a la Unidad de Pruebas Funcionales de Urología y las interconsultas remitidas desde consultas externas, puesto que estas últimas son gestionadas dentro de éste módulo. El 32,5\% de la interconsultas por PSA fueron solicitadas como preferentes (Tabla 3).

Los días de respuesta a las IC fue de media de 1,45 días con desviación típica de 1,65 días (mínimo de en las siguientes 24 horas y máximo al $10^{\circ}$ día). Se respondieron el mismo día un 36,4\% (n=142 ), al día siguiente el 31,5\% (n=129) y el $12 \%(n=49)$ a las 48 horas. Y cuando las dividimos en función de la prioridad con la que fue solicitada: Normales media de 1,72 días (Desviación típica de 1,76 días), preferentes media de 1,25 días (Desviación típica de 1,54) y urgentes media de 0,42 días (Desviación típica de 1,02). Véase de forma más detallada (Tabla 4).

Según los criterios establecidos, para clasificar los documentos por nivel de adecuación, fueron catalogadas como inapropiadas al 32,9\% de las IC $(n=135)$, siendo el $71 \%$ de éstas correspondientes a servicios médicos $(\mathrm{p}=0,000)$.

Fueron clasificadas en grado 1 el 44,6\% ( $n=183)$, grado 2 el 20\% ( $n=82)$ y grado 3 el 2,4\% $(n=10) . ~ S i$ tenemos en cuenta el Grado 2 y 3, se solicitaron como IC normales el 20\%, 16,7\% preferentes y 50\% de las urgentes. Si estudiamos el grado con la que fue clasificada la IC con el servicio de procedencia ver (Tabla 5)

Tabla 3. Tipo de interconsulta

\begin{tabular}{lcccc}
\hline & Normal & Preferente & Urgente & Total \\
\hline Servicios médicos & 190 & 76 & 43 & $309(75,4 \%)$ \\
Servicios quirúrgicos & 70 & 26 & 5 & $101(24,6 \%)$ \\
Total & $260(63,3 \%)$ & $102(24,8 \%)$ & $48(11,7 \%)$ & \\
\hline
\end{tabular}

Chi Cuadrado de Pearson: 0,000 
Tabla 4. Días espera. Respuesta

\begin{tabular}{|c|c|c|c|c|c|c|c|c|c|c|}
\hline TIPO IC & 0 & 1 & 2 & 3 & 4 & 5 & 6 & 7 & 10 & Total \\
\hline Normal & 67 & 83 & 41 & 29 & 18 & 15 & 3 & 3 & 1 & 260 \\
\hline Preferente & 37 & 40 & 8 & 5 & 7 & 3 & 0 & 2 & 0 & 102 \\
\hline \multirow[t]{2}{*}{ Urgente } & 38 & 6 & 4 & 0 & 0 & 0 & 0 & 0 & 0 & 47 \\
\hline & $\begin{array}{c}142 \\
(34,6 \%)\end{array}$ & $\begin{array}{c}129 \\
(31,5 \%)\end{array}$ & $\begin{array}{c}52 \\
(14 \%)\end{array}$ & $\begin{array}{c}37 \\
(9 \%)\end{array}$ & $\begin{array}{c}25 \\
(6,1 \%)\end{array}$ & $\begin{array}{c}19 \\
(4,6 \%)\end{array}$ & $\begin{array}{c}3 \\
(0,7 \%)\end{array}$ & $\begin{array}{c}5 \\
(1,2 \%)\end{array}$ & $\begin{array}{c}1 \\
(0,2 \%)\end{array}$ & $\begin{array}{c}410 \\
(100 \%)\end{array}$ \\
\hline
\end{tabular}

Tabla 5. Nivel de adecuación

\begin{tabular}{lccccc}
\hline Servicio & $\mathbf{0}$ & $\mathbf{1}$ & $\mathbf{2}$ & $\mathbf{3}$ & Total \\
\hline $\begin{array}{l}\text { Médico } \\
\text { \% del servicio }\end{array}$ & 94 & 144 & 62 & 9 & 309 \\
& $(30,4)$ & $(46,6)$ & $(20,1)$ & $(2,9)$ & $(100)$ \\
$\begin{array}{l}\text { Quirúrgico } \\
\text { \% del servicio }\end{array}$ & 41 & 39 & 20 & 1 & 101 \\
Total & 135 & $183,6)$ & $(19,8)$ & $(1)$ & $(100)$ \\
\% del total IC & $(32,9)$ & $(44,6 \%)$ & $(20 \%)$ & $(2,4 \%)$ & \\
\hline
\end{tabular}

Chi cuadrado de Pearson $p<0,2$

La actitud que tomó el Urólogo o Residente de Urología fueron las siguientes: se remitió a Consultas Externas de Urología al 10,5\% del total de las IC (83\% de éstas de servicios médicos), al Centro de Atención Especializada al 7,8\% (62,5\% correspondientes a Servicios médicos), se solicitó pruebas complementarias al 7,8\%, se remitió al Pruebas funcionales de Urología, para la realización de alguna prueba complementaria a 61 pacientes $(14,9 \%)$, se presentó en Sesión Clínica de Urología al 5,6\% de los pacientes ( $n=23$ ) (dentro de éstas $87 \%$ de servicios médicos), se trasladó al Servicio de Urología a 2 pacientes $(0,5 \%)$ y se tomó alguna otra actitud terapéutica en el 19,6\%, estos resultados tuvieron una significación de $\mathrm{p}=0,000$. Por tipo de IC: no se tomó ninguna actitud en el $31,5 \%$ de las IC normales, 34,3\% de las preferentes y $35,4 \%$ de las urgentes. Y la actitud que se tomó con el enfermo, no tuvo diferencias significativas según el médico respondedor $(\mathrm{p}=0,016)$.

\section{DISCUSIÓN}

El documento de interconsulta en formato papel es la herramienta utilizada en la comunicación entre ambos especialistas, normalizado en nuestro medio, consiste en una doble hoja autocopiativa con 2 espacios diferenciados: uno para la información aportada por el derivante y otro para la respuesta del consultor. Una de las copias queda en la historia del paciente y la otra es para el servicio consultado.
La importancia de reflejar en este documento una correcta y completa historia médica facilita y orienta sobre el motivo que ha causado la consulta. De igual forma, es fundamental que la actuación del otro especialista llegue al médico para integrar y coordinar la asistencia, para que ésta sea efectiva.

Adicionalmente, una de las cargas de trabajo de un Servicio de Urología es la respuesta a las consultas de especialidades médicas o quirúrgicas para el planteamiento global y solución de problemas, en ocasiones multidisciplinares.

Dos estudios nacionales ${ }^{2,3}$ han analizado la interconsulta en los hospitales, en servicios de Medicina Interna y ambos revelan que, ésta es frecuente (4 casos por cada 100), interesa a individuos con una edad media elevada (65 a 70 años) y atiende a un subgrupo específico de enfermos con patología múltiple, cuya estancia media y mortalidad es alta.

Existe una gran variabilidad entre los médicos respecto al número de consultas que realizan a otros especialistas, por lo que resulta difícil valorar la necesidad o conveniencia de las mismas. Factores como la edad, los años trabajados o el grado de formación del médico se han planteado como factores que podrían modificar esta tasa, aunque los datos no son definitivos ${ }^{2,4}$. La presión asistencial también se ha sugerido como probable factor de influencia. Resultando que, una alta tasa de consultas puede suponer una demanda inapropiada, generando una carga asistencial significativa, aunque por otro lado una baja tasa puede plantear dudas sobre la calidad asistencial recibida.

En nuestro estudio, observamos un alto porcentaje en cuanto a la constancia de los datos de filiación (96,3\%), ligeramente inferior en la descripción de la sintomatología y considerados bajos en la exploración (sin diferencias significativas entre servicios médicos ni quirúrgicos). Y esto último, quizás debido al sobreentendimiento de que será el Urólogo quién lo realice. 
El alto porcentaje de IC urgentes y preferentes consideradas inapropiadas (mayor a 1/3 de cada una de éstas) posiblemente se deba a varios factores: en muchos casos existe una valoración diferente de la urgencia entre el médico que solicita la IC y el que la responde y por otro lado la urgencia de la IC puede ser clasificada como urgencia verdadera, debida a una situación médica grave e inestable, inducida por la intranquilidad del médico responsable del enfermo, urgencia administrativa, originada por una valoración y la provocada por un tiempo de respuesta habitual, considerado por los consultores como prolongado. Aunque sólo disponemos una referencia previa ${ }^{3}$, donde sólo el 3\% resultaban inapropiadas, estamos ante un estudio de 100 IC realizada hace 14 años, en un hospital que no pertenece a nuestro medio.

Respecto a la actitud que tomó el Urólogo o Residente de Urología, quizás hubo una infrautilización de la Unidad de Pruebas funcionales de Urología (14,9\% del total de los pacientes), que puede estar asociado a la escasez de recursos destinados a dicha Unidad.

Una importante limitación de este trabajo es que se trata de un estudio retrospectivo descriptivo, aunque existe un "doble ciego" ni el médico consultor, el facultativo responsable del servicio de urología, el paciente ni ninguno de los miembros del personal implicado en la tramitación de la IC eran conocedores de la realización del presente estudio.

Hasta el momento y bajo nuestro conocimiento sólo existe un estudio realizado en el ámbito de las IC realizadas al Servicio de Urología en un Hospital de tercer nivel, de Batista Miranda JE et al. en el año 1994, con características similares. Y creemos que la realización de estudios para profundizar en el conocimiento y problemática de la IC puede ser de gran utilidad, permitiendo identificar problemas propios de cada centro, incrementar entre los servicios e incluso establecer criterios comunes para las IC. Todo ello permitirá plantear estrategias para incrementar la calidad de la asistencia a los pacientes y mejorar diversos aspectos de la actividad hospitalaria.

\section{REFERENCIAS}

1. Rubio Arribas V, Rodríguez Ibáñez ML, Sampedro Martínez E, Victores Benavente C, Alechiguerra García A, Barrio Gamarra JL. Evaluación de la calidad de comunicación entre niveles asistenciales mediante el documento de interconsulta. Aten Primaria. 2000;26:681-684.

2. Wilkin D, Smith A. Explaining variation in general practitioner referral to hospital. Fam Pract. 1987;4(3):160-169.

3. Batista Miranda JE, Errando Smet C, Ponce de Leon Roca J, Caicedo Caicedo P, Fariña Pérez L. Urologic interconsultation in a general hospital: analysis of 100 consecutive consultations. Arch Esp Urol. 1994;47(3):307-311.

4. Díaz Peromingo JA, Grandes Ibáñez J, Castro Paredes B, Cabezas Agrícola JM, de la Iglesia Martínez R, Baeza Barrantes FJ. Estudio de la interconsulta en un servicio de Medicina Interna. Rev Clin Esp 1999;199:66-72.

5. Monte Secades R, Rabuñal Rey R, Rigueiro Veloso MT, García Pais MJ, Casariego Vales E, Guerrero Lombardía J. Papel del internista como consultor de servicios quirúrgicos. Rev Clin Esp 2004;204(7):345-350.

Correspondencia autor: Patricio Navarro Medina Servicio de Urología. Complejo Hospitalario Universitario Insular Materno Infantil de Gran Canaria

Pza. del Doctor Pasteur, s/n

35016 Las Palmas de Gran Canaria. Tel.: 928444000

E-mail autor: patricionm@terra.es

Información artículo: Original

Trabajo recibido: octubre 2008

Trabajo aceptado: noviembre 2008 